Received: 2017.05 .16

Accepted: 2017.06.23

Published: 2017.12.30

\title{
Analysis of CYP2C19 Genetic Polymorphism in a Large Ethnic Hakka Population in Southern China
}

Authors' Contribution: Study Design A Data Collection B Statistical Analysis C Data Interpretation D Manuscript Preparation E Literature Search F Funds Collection G
BCDEF 1,2 Zhixiong Zhong*

BCDEF 2,3 Jingyuan Hou*

BC 1 Bing Li

$B C D 1$ Qifeng Zhang

$B C D$ 2,3 Sudong Liu

BCD 1 Cunren Li

BC 1 Zhidong Liu

BC 1 Min Yang

BC 1 Wei Zhong

ABCDEFG 2,3 Pingsen Zhao
1 Center for Cardiovascular Diseases, Meizhou People's Hospital, Huangtang Hospital, Meizhou Hospital Affiliated to Sun Yat-sen University, Meizhou, Guangdong, P.R. China

2 Center for Precision Medicine, Meizhou People's Hospital, Huangtang Hospital, Meizhou Hospital Affiliated to Sun Yat-sen University, Meizhou, Guangdong, P.R. China

3 Clinical Core Laboratory, Meizhou People's Hospital, Huangtang Hospital, Meizhou Hospital Affiliated to Sun Yat-sen University, Meizhou, Guangdong, P.R. China
Corresponding Author: Source of support:

Background:

Material/Methods:

Results:

Conclusions:

MeSH Keywords:

Full-text PDF:
* Contributed equally to this work

Pingsen Zhao, e-mail: zhaopingsen01@163.com, zhaopingsen@hotmail.com

This study was supported by the Natural Science Foundation of Guangdong Province, China (Grant No.: 2016A030307031 to Dr. Pingsen Zhao), the Natural Science Foundation of Guangdong Province, China (Grant No.: 2014 A030307042 to Dr. Pingsen Zhao), the National Key Research and Development Program of China (Grant No.: 2016YFD0050405 to Dr. Pingsen Zhao), the Medical Scientific Research Foundation of Guangdong Province, China (Grant No.: A2016306 to Pingsen Zhao), and the Key Scientific and Technological Project of Meizhou People's Hospital, Guangdong Province, China (Grant No.: MPHKSTP-20170102 to Pingsen Zhao)

Cytochrome P450 (CYP) 2 C19 is an enzyme involved in the bioactivation of various important therapeutic drugs, from pro-drugs to an active inhibitor of platelet action. Variants in the CYP2C19 gene influence the pharmacokinetics and clinical response to antiplatelet drugs such as clopidogrel; however, there is no available data about the genetic variation of CYP2C19 in the Hakka population in China.

A total of 6686 unrelated participants (ages 17-98 years) of self-reported Hakka ancestry admitted at an inpatient department in a hospital in southern China were successfully genotyped by the gene chip platform.

The identified allele frequencies were CYP2C19*1 (64.33\%), *2 (31.06\%) and *3 (4.61\%). The major prevalent genotype combinations were CYP2C19 ${ }^{*} 1 /{ }^{*} 1(41.73 \%)$ and ${ }^{*} 1 /{ }^{*} 2(39.65 \%)$. The distribution of CYP2C19 phenotypes was divided into extensive metabolizers (EM) (41.73\%), intermediate metabolizers (IM) (45.21\%), and poor metabolizers (PM) (13.06\%). In the Hakka population, frequencies of the CYP2C19 *2 and * 3 variants were observed to be close to those previously identified in Chinese and several other Asian populations.

Our study is the first to report on CYP2C19 polymorphisms in the Hakka population, and may help to optimize pharmacotherapy effectiveness by providing personalized medicine to this ethnic group in the near future.

\section{China • Pharmacogenetics • Polymorphism, Genetic}

https://www.medscimonit.com/abstract/index/idArt/905337

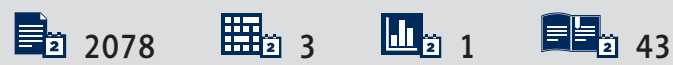




\section{Background}

Advances in personalized medicine have been notable, as the role of inheritance in variable drug response are being unraveled and pharmacogenetics are being applied to develop individual-specific therapies. Inter-individual variability in drug dosage response or lack of response to a drug, as well as drug toxicity, can maximize drug efficacy and avoid adverse drug reactions and therapeutic failures [1,2]. The availability of genetic variation in genes encoding for drug metabolizing enzymes is a key element of individualized care. Cytochrome P450 (CYP) is a superfamily of phase I metabolizing enzymes, which play a critical role in biosynthesis and degradation of drugs, endogenic substances, and toxins [3]. Over the past few years, a variety of cytochrome P450 (CYP) enzymes that participate in phase I reactions of drug metabolism, including CYP1A2, CYP2C9, CYP2C19, and CYP2D6, have been described [4,5].

CYP2C19 is an important member of the CYP450 superfamily, which plays a substantial role in the metabolism of approximately $10 \%$ of commonly prescribed drugs such as proton pump inhibitors, antipsychotics, antidepressants, and clopidogrel [6]. Like many other CYP450 superfamily members, inter-individual variability in response to CYP2C19 substrate can be explained satisfactorily by factors such as highly polymorphic of CYP2C19 genes, and individuals can be classified into predicted phenotypes of poor metabolizers (PM), intermediate metabolizers (IM), extensive metabolizers (EM), or ultrarapid metabolizers (UM) $[7,8]$. Currently, at least 34 known variant alleles and numerous subvariants have been identified within the CYP2C19 gene. Many studies have focused on the association between genetic polymorphisms and clopidogrel resistance.

Multiple mechanisms have been proposed for the clopidogrel antiplatelet response variability, and substantial evidence has linked the CYP2C19 genotype with the clinical response among clopidogrel-treated patients. Accumulating evidence has demonstrated that both heterozygotes and homozygotes for loss-offunction alleles, particularly CYP2C19² (c.681G>A; rs4244285) and CYP2C19*3 (c.636G>A; rs4986893), are responsible for reduced activation of clopidogrel and an increased rate of recurrent cardiovascular events [9-11]. In 2010, the US Food and Drug Administration (FDA) added a black-box warning about the diminished effectiveness of clopidogrel, which suggests that individuals with poor metabolizer genotypes may be at increased risk for adverse cardiovascular outcomes [12]. The use of CYP2C19 genetic testing to guide antiplatelet therapy in these high-risk patients with cardiovascular diseases appears to be an appealing strategy. Moreover, the polymorphism of CYP2C19 varies considerably with both geographical location and ethnic group [13]. To date, numerous investigations of the differences in allele frequencies of the CYP2C19 gene in various ethnic populations worldwide have been reported.
Prior knowledge of the genetic variants that are present in a population may facilitate the revision and optimization of existing medication choices and doses. China is a multi-ethnic country with 55 ethnic minorities. Hakka is an intriguing Han Chinese ethnic group that has a population living in southern China, which is characteristic of their unique culture with similarities to northern Han populations. More than $95 \%$ of people who live in the Meizhou region of Guangdong province are Hakka and possess some unique features in culture, language, diet, lifestyle, and environment [14]. To the best of our knowledge, no data is available on CYP2C19 polymorphism in the Hakka population. The aim of this study was to describe CYP2C19 genetic variants in Hakka people in our population. The present study investigated the incidences of different CYP2C19 mutant allele ( ${ }^{\star} 2$ and ${ }^{*} 3$ ) frequencies in the Hakka population attending an inpatient department of a hospital in southern China and to determine genotype frequencies for these mutations. The CYP2C19 genotypes were then classified into phenotypes. We also compared genetic polymorphisms of CYP2C19 with previous observations in other ethnic groups.

\section{Material and Methods}

\section{Study subjects}

This was a retrospective study. The study protocol conformed to the principles of the Declaration of Helsinki, and was approved by the Ethics Committee of Meizhou People's Hospital. In total, 6686 unrelated subjects (ages 17-98 years) who visited Meizhou People's Hospital, Guangdong, Republic of China, were enrolled in the study from November 2015 to April 2017. Written informed consent was obtained from all participants prior to enrollment in the study.

\section{DNA extractions}

Blood samples from a peripheral vein were collected from each participant and stored in 6-ml evacuated vacuum tubes containing ethylenediaminetetraacetic acid (EDTA). Genomic DNA extraction was carried out using the Puregene Blood Core Kit C (Qiagen, Germantown, MD, USA) following the manufacturer's instructions, and DNA concentration was quantified using the Nanodrop 2000 ${ }^{\mathrm{TM}}$ Spectrophotometer (ThermoFisher Scientific, Waltham, MA). Only quality DNA (A260/280 ratio >1.7) was stored at $-80^{\circ} \mathrm{C}$ until analysis.

\section{Polymerase chain reaction and DNA sequencing}

The single-nucleotide polymorphisms CYP2C19*2 and CYP2C19*3 were genotyped using a commercially available kit (BaiO Technology Co, Ltd, Shanghai, China). Polymerase chain reaction (PCR) was performed according to the following 


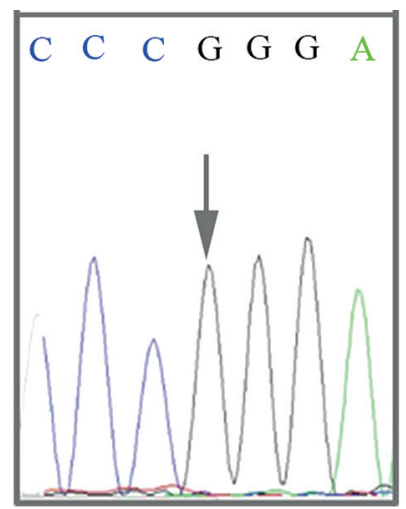

GG

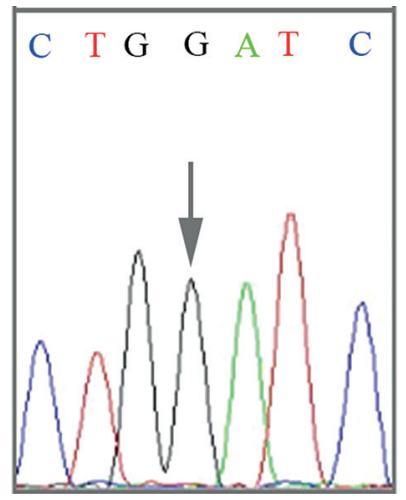

GG

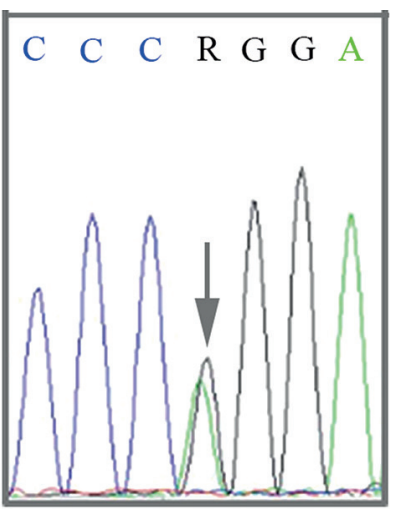

$\mathrm{G} / \mathrm{A}$

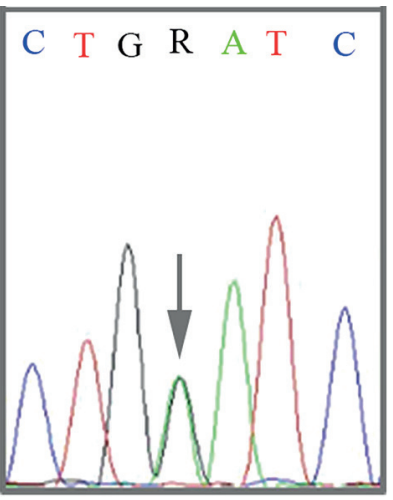

$\mathrm{G} / \mathrm{A}$

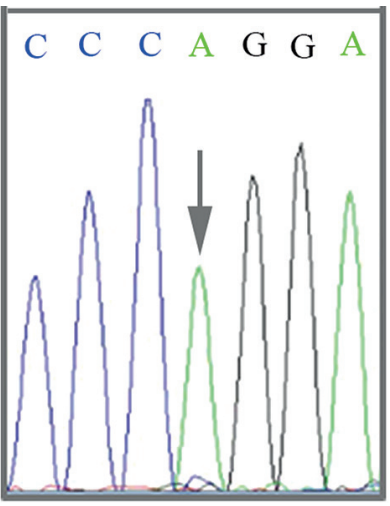

$A A$

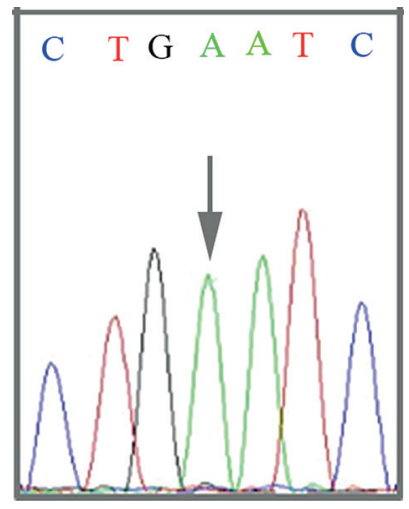

$\mathrm{AA}$
CYP2C19*2

(c.681G>A)

CYP2C19*3 (c.636G $>A)$

Figure 1. Sequence chromatogram of CYP2C19*2(c.681G>A; rs4244285) (up) and CYP2C19*3 (c.636G >A; rs4986893) (down). SNPs are indicated by arrows.

protocol: $50^{\circ} \mathrm{C}$ for $5 \mathrm{~min}$, pre-denaturation at $94^{\circ} \mathrm{C}$ for $5 \mathrm{~min}$, followed by 35 cycles of denaturing at $94^{\circ} \mathrm{C}$ for $25 \mathrm{~s}$, annealing at $48^{\circ} \mathrm{C}$ for $40 \mathrm{~s}$, and extension at $72^{\circ} \mathrm{C}$ for $30 \mathrm{~s}$, and a final elongation at $72^{\circ} \mathrm{C}$ for $5 \mathrm{~min}$. The amplification products were subsequently dispensed into a hybridization reaction chamber to hybridize reactions. BaiO Array Doctor Version 2.0 (BaiO Technology Co, Ltd, Shanghai, China) software and BaiO ${ }^{\circledR}$ BE-2.0 (BaiO Technology Co, Ltd, Shanghai, China) software were utilized to analyze the images of the hybridization of the amplification products with the gene probes according to the instructions of the manufacturer.

Sanger sequencing was performed to confirm the different allelic variants of CYP2C19* 2 and CYP2C19*3. Sequencing of the purified products using reverse primers was conducted with the sequencing kit according to the instructions of the manufacturer (SinoMDgene Technology Co., Ltd, Beijing, China). The DNA sequencing was performed on an ABI 3500xL Genetic Analyzer (Applied Biosystems). The sequencing results were assembled using ABI PRISM sequencing analysis software version 5.4 (Applied Biosystems). The chromatograms of different SNP variants using reverse primers for sequencing are presented in Figure 1.

\section{Statistics}

SPSS statistical software version 19.0 was used for data analysis. Chi-square and Fisher's exact tests were used to compare the allele and genotype frequencies and descriptive analysis was used to compare allele frequencies between the Hakka population and published data of other ethnic groups. A value of $P<0.05$ was considered as statistically significant.

\section{Results}

Genotype and allelic frequencies of CYP2C19 * 1 , *2, *3 in 6686 subjects of the Hakka population attending an inpatient department of a hospital in southern China were successfully obtained and are shown in Tables 1 and 2. The allelic frequency of CYP2C19*2 was 0.31 , with 652 individuals homozygous for this defective allele resulting in a frequency of $9.57 \%$ for the ${ }^{*} 2 /{ }^{*} 2$ genotype. The frequency of CYP2C $19{ }^{*} 3$ allele was 0.05 , with $0.35 \%$ of the Hakka population harboring the homozygous ${ }^{*} 3 /{ }^{*} 3$ genotype; therefore, the wild-type allele CYP2C19*1 exhibited the highest frequency at 0.64 . The observed frequencies of 
Table 1. Genotype frequencies of CYP2C19 in 6,686 subjects of Hakka ethnic population.

\begin{tabular}{|c|c|c|c|c|}
\hline \multicolumn{2}{|c|}{ CYP2C19 } & \multirow{2}{*}{$\begin{array}{l}\text { Observed } \\
\text { number }\end{array}$} & \multirow{2}{*}{$\begin{array}{c}\text { Expected } \\
\text { number }\end{array}$} & \multirow{2}{*}{ Frequency (\%) } \\
\hline Phenotypes & Genotype & & & \\
\hline Extensive metabolizer & ${ }^{*} 1 /{ }^{*} 1$ & 2,790 & 2,767 & 41.73 \\
\hline \multirow{2}{*}{ Intermediate metabolizer } & ${ }^{*} 1 /{ }^{*} 2$ & 2,651 & 2,672 & 39.65 \\
\hline & ${ }^{*} 1 /{ }^{*} 3$ & 372 & 396 & 5.56 \\
\hline \multirow{3}{*}{ Poor metabolizer } & ${ }^{*} 2 /{ }^{*} 2$ & 652 & 645 & 9.75 \\
\hline & ${ }^{*} 2 /{ }^{*} 3$ & 198 & 191 & 2.96 \\
\hline & ${ }^{*} 3 /{ }^{*}$ & 23 & 14 & 0.35 \\
\hline Total & & 6,686 & & 100.00 \\
\hline
\end{tabular}

Table 2. Ethnic variation of $\mathrm{CYP} 2 \mathrm{C} 19\left({ }^{\star} 1,{ }^{\star} 2,{ }^{*} 3\right)$ in the present study and previous studies.

\begin{tabular}{|c|c|c|c|c|c|}
\hline \multirow{2}{*}{ Populations } & \multirow{2}{*}{ Number } & \multicolumn{3}{|c|}{ Alleles frequency of CYP2C19 } & \multirow{2}{*}{ Reference } \\
\hline & & $*_{1}$ & *2 & *3 & \\
\hline Hakka & 6,686 & 0.6433 & 0.3106 & 0.0461 & \\
\hline Chinese-Dai & 193 & 0.66 & 0.30 & 0.03 & 33 \\
\hline Chinese Li & 100 & 0.74 & 0.25 & 0.01 & 25 \\
\hline Chinese-Han & 101 & 0.56 & 0.37 & 0.07 & 34 \\
\hline Koreans & 103 & 0.67 & 0.21 & 0.12 & 22 \\
\hline Japanese & 1,003 & 0.59 & 0.30 & 0.11 & 24 \\
\hline Vietnamese & 90 & 0.62 & 0.24 & 0.14 & 22 \\
\hline Thai & 1,051 & 0.63 & 0.27 & 0.10 & 30 \\
\hline Malaysian & 54 & 0.72 & 0.23 & 0.05 & 21 \\
\hline Turkish & 404 & 0.88 & 0.12 & 0.00 & 35 \\
\hline Saudi Arabians & 97 & 0.85 & 0.15 & 0.13 & 40 \\
\hline Iranians & 140 & 0.56 & 0.24 & 0.20 & 44 \\
\hline Mexican & 238 & 0.77 & 0.08 & - & 23 \\
\hline Macedonian & 184 & 0.65 & 0.14 & - & 22 \\
\hline Swedish & 175 & 0.77 & 0.23 & 0.00 & 36 \\
\hline Russian & 290 & 0.88 & 0.11 & 0.00 & 32 \\
\hline Italian & 360 & 0.89 & 0.11 & 0.00 & 28 \\
\hline Bolivian & 778 & 0.92 & 0.08 & 0.00 & 37 \\
\hline Faroese & 312 & 0.97 & 0.03 & 0.00 & 38 \\
\hline Tanzanian & 251 & 0.81 & 0.18 & 0.01 & 41 \\
\hline Ethiopian & 114 & 0.84 & 0.14 & 0.02 & 42 \\
\hline Zimbabwean & 84 & 0.87 & 0.13 & 0.00 & 43 \\
\hline
\end{tabular}

This work is licensed under Creative Common Attribution NonCommercial-NoDerivatives 4.0 International (CC BY-NC-ND 4.0)

\section{9}

Indexed in: [Current Contents/Clinical Medicine] [SCI Expanded] [ISI Alerting System] [ISI Journals Master List] [Index Medicus/MEDLINE] [EMBASE/Excerpta Medica] [Chemical Abstracts/CAS] [Index Copernicus] 
Table 3. Alleles and genotypes frequencies for the two SNPs in the Hakka population.

\begin{tabular}{|c|c|c|c|c|c|c|}
\hline \multirow{2}{*}{ Gene } & \multicolumn{4}{|c|}{ Genotype } & \multicolumn{2}{|c|}{ Gene frequency } \\
\hline & GG & GA & AA & Total & G & A \\
\hline $\begin{array}{l}\text { CYP2C19*2 } \\
\text { (rs4244285) }\end{array}$ & 3,185 (47.64) & $2,849(42.61)$ & $652 \quad(9.75)$ & 6,686 & 9,219 (68.94) & $4,153(31.06)$ \\
\hline $\begin{array}{l}\text { CYP2C19*3 } \\
\text { (rs4986893) }\end{array}$ & $6,093(91.13)$ & $570 \quad(8.53)$ & $23 \quad(0.34)$ & 6,686 & 12,756 (95.39) & $616 \quad(4.61)$ \\
\hline
\end{tabular}

CYP2C19 genotypes in the Hakka ethnic group were in HardyWeinberg equilibrium $\left(\chi^{2}=7.62, P>0.05\right)$.

The frequencies and genotypes of CYP2C19*2 and CYP2C19*3 alleles in Chinese Hakka subjects are summarized in Table 3. The frequency of mutant allele CYP2C19*2 was almost 6-fold higher than that of mutant allele CYP2C19*3. Among the 6686 subjects, there were 3185 (47.64\%) with wild-type homozygous, 2849 (42.61\%) with heterozygous, and 652 (9.75\%) with mutant homozygous CYP2C19*2. There were 6093 (91.13\%) with wild-type homozygous, 570 (8.53\%) with heterozygous, and $23(0.34 \%)$ with mutant homozygous CYP2C19*3. We divided these 6686 subjects into 3 phenotypes based on CYP2C19*2 and CYP2C19*3 genotypes in the present study (Table 1). The normal metabolizer genotype was the extensive metabolizer (EM; $41.73 \%)$, followed by intermediate metabolizer (IM; $45.21 \%)$ and poor metabolizer (PM; $13.06 \%)$ genotypes.

To ensure accuracy, 300 samples were re-genotyped by Sanger sequencing. We obtained the same genotyping result for each selected sample by both methods (Gene chip method and Sanger sequencing).

\section{Discussion}

Numerous lines of evidence strongly suggest that inter-individual, ethnic, and racial differences in drug metabolism are due to polymorphic expression of metabolizing enzymes, especially cytochrome P450 (CYP) $[15,16]$. Recent data demonstrate significant alteration in the enzyme activity result in variable drug responses and increased rates of thrombotic events in patients harboring hepatic cytochrome gene variants [10,1719]. CYP2C $19^{*} 2$ and CYP2C19*3 alleles, which result in the aberrant splicing and a premature stop codon, respectively, have been extensively studied in populations of different ethnicities and geographic origin [20-23]. Since CYP2C19*2 and *3 cover $>90 \%$ of the poor metabolism population, the mutation genotype contained ${ }^{*} 1 /{ }^{*} 2,{ }^{*} 1 /{ }^{*} 3,{ }^{*} 2 /{ }^{*} 2,{ }^{*} 2 /{ }^{*} 3$, and ${ }^{*} 3 /{ }^{*} 3$. The allele frequency of Asian backgrounds has been reported to have a substantially greater prevalence of CYP2C19 polymorphisms associated with loss-of-function of this enzyme when compared to other racial groups [22,24,25]. For the first time, we analyzed the frequency of CYP2C19 polymorphisms in the Hakka population and compared these results with racial populations from different continents. All genotype frequencies for both CYP2C19*2 and CYP2C19*3 alleles were consistent with Hardy-Weinberg equilibrium.

We compared the allele frequencies estimated for $\mathrm{CYP} 2 \mathrm{C} 19^{*} 2$ and CYP2C19*3 with respect to previously published reports in other ethnic populations (Table 2). Previous studies have shown that the allelic frequency of CYP2C19*2 in Asian populations are up to $30.0 \%$, and are $15.0-17.0 \%$ in Europeans and blacks [26-28]. The allelic frequencies of CYP2C19*2 in west Asian, European, South American, Scandinavian, and AfricanAmerican populations are relatively low [29-32]. Compared to other ethnicities, the CYP2C19 loss-of-function alleles are notably higher in Asian populations. Overall, the allelic frequency of CYP2C19*2 of the Hakka subjects (31.06\%) are closer to that of Chinese-Dai (30\%), but are in between that of populations from Chinese $\mathrm{Li}(25 \%)$ and Chinese-Han ethnic groups (37\%) $[25,33,34]$. The frequency of the CYP2C19*2 in our study was similar to that in studies of other Asian populations (Table 2).

On the other hand, the CYP2C19*3 allele was found with a frequency of 0.0461 in the Hakka population, which is consistent with findings within East Asian populations (Table 2). CYP2C19*3 is relatively common in Asians and accounted for the remaining defective alleles in Asian PMs after genotyping for $\mathrm{CYP} 2 \mathrm{C}^{2}{ }^{*} 2$. In contrast, previous reports suggested that CYP2C19*3 was present at a low frequency or nearly absent in Turkish, Swedish, Russian, Italian, Bolivian, Faroese, Tanzanian, Ethiopian, and Zimbabwean populations [32,35-38]. The prevalence of $\mathrm{CYP} 2 \mathrm{C} 19^{*} 3$ presented in our study is consistent with previous reports on the Chinese population, but lower than that of Japanese, Koreans, Vietnamese, and Thai populations [22,39]. Despite this, no statistically significant difference in frequency of CYP2C19*3 was found between the Hakka and the populations mentioned above. In addition, more attention should be paid to populations that have a high frequency of the CYP2C19 lossof-function alleles, especially in China, because people with the variants CYP2C19*2 or CYP2C19*3 can have abnormal in metabolism of drugs such as clopidogrel, with adverse drug reactions. 
As described earlier, several studies have provided evidence that CYP2C19*2 and CYP2C19*3 alleles are consistently associated with adverse cardiovascular events. Therefore, clinical use of the CYP2C19 genotype as a predictive biomarker for personalized therapy is crucial, and can be classified into the 3 following metabolizer phenotypes: poor metabolizers (PM), intermediate metabolizers (IM), and extensive metabolizers (EM). In the present study, the frequencies of extensive metabolizers and intermediate metabolizers were observed to be comparable, although the proportion of intermediate metabolizers (45.21\%) was found at a slightly higher frequency than extensive metabolizers (41.73\%). The most important mutated allele was $\mathrm{CYP} 2 \mathrm{C} 19^{*} 2$, which was predominantly responsible for poor metabolizers and accounted for $86.03 \%$ of the study population. The frequencies of the mutant CYP2C19 alleles and genotypes showed large inter-ethnic differences. As with previous studies, poor metabolizers are significantly more frequent (12-23\%) in the East Asian population, whereas the prevalence of poor metabolizers is estimated to be lower in West Asian, Europeans, South Americans, Scandinavians, and Africans [22,40-43]. In this study, we observed that the prevalence of the poor metabolizers' phenotype is $13.06 \%$, which is similar to that of other Asian populations.

\section{References:}

1. Shastry BS: Pharmacogenomics and pharmacoepigenomics in pediatric medicine. Methods Mol Biol, 2014; 1175: 671-85

2. Ahmadi KR, Weale ME, Xue ZY et al: A single-nucleotide polymorphism tagging set for human drug metabolism and transport. Nat Genet, 2004; 37 84-89

3. Scott SA, Sangkuhl K, Gardner EE et al: Clinical Pharmacogenetics Implementation Consortium guidelines for cytochrome P450-2C19 (CYP2C19) genotype and clopidogrel therapy. Clin Pharmacol Ther, 2011; 90: 328-32

4. Mega JL, Close SL, Wiviott SD et al: Cytochrome P450 genetic polymorphisms and the response to prasugrel: Relationship to pharmacokinetic, pharmacodynamic, and clinical outcomes. Circulation, 2009; 119: 2553-60

5. Jang JS, Cho KI, Jin HY et al: Meta-analysis of cytochrome P450 2C19 polymorphism and risk of adverse clinical outcomes among coronary artery disease patients of different ethnic groups treated with clopidogrel. Am J Cardiol, 2012; 110: 502-8

6. Scott SA, Sangkuhl K, Shuldiner AR et al: PharmGKB summary: Very important pharmacogene information for cytochrome P450, family 2, subfamily C, polypeptide 19. Pharmacogenet Genomics, 2012; 20: 520-23

7. Hulot JS, Bura A, Villard E et al: Cytochrome P450 2C19 loss-of-function polymorphism is a major determinant of clopidogrel responsiveness in healthy subjects. Blood, 2006; 108: 2244-47

8. Mega JL, Simon T, Collet JP et al: Reduced-function CYP2C19 genotype and risk of adverse clinical outcomes among patients treated with clopidogrel predominantly for PCI: A meta-analysis. JAMA, 2010; 304: 1821-30

9. Hulot JS, Collet JP, Silvain J et al: Cardiovascular risk in clopidogrel-treated patients according to cytochrome P450 2C19*2 loss-of-function allele or proton pump inhibitor coadministration: A systematic meta-analysis. J Am Coll Cardiol, 2010; 56: 134-43

10. Marian AJ: Cytochrome $\mathrm{P}-450$ polymorphisms and response to clopidogrel. Curr Atheroscler Rep, 2009; 360: 2250-51

11. Sorich MJ, Vitry A, Ward MB et al: Prasugrel vs. clopidogrel for cytochromeP450 2C19-genotyped subgroups: Integration of the TRITON-TIMI38 trial data. J Thromb Haemost, 2010; 8: 1678-84

\section{Conclusions}

For the first time, we describe the genetic polymorphism of CYP2C19 (CYP2C19*2 and *3) in the Hakka ethnic population. The CYP2C19 allele and genotype frequencies were also compared to other populations. In particular, most of the previous studies were conducted with relatively low numbers of individuals. In comparison, a large sample size of 6686 subjects was genotyped in our study and the results may deepen our understanding of the basic genetic profile of CYP2C19 in the Hakka population. The results of the present study offer a preliminary basis for more rational use of drugs that are substrates for CYP2C19 in Hakka subjects. Further studies should focus on obtaining sufficient data on allelic frequencies related to drug response for their implementation in clinical practice.

\section{Conflicts of interest}

None.

12. Society for Cardiovascular Angiography and Interventions; Society of Thoracic Surgeons; Writing Committee Members, Holmes DR Jr., Dehmer GJ, Kaul S et al: ACCF/AHA Clopidogrel clinical alert: Approaches to the FDA "boxed warning": a report of the American College of Cardiology Foundation Task Force on Clinical Expert Consensus Documents and the American Heart Association. Circulation, 2010; 122: 537-57

13. Shuldiner DAR, O'Connell DJR, Bliden MKP et al: Association of cytochrome P450 2C19 genotype with the antiplatelet effect and clinical efficacy of clopidogrel therapy. JAMA, 2009; 302: 849-57

14. Wang WZ, Wang CY, Cheng YT et al: Tracing the origins of Hakka and Chaoshanese by mitochondrial DNA analysis. Am J Phys Anthropol, 2010; 141: $124-30$

15. Holmes MV, Perel P, Shah T et al: CYP2C19 genotype, clopidogrel metabolism, platelet function, and cardiovascular events: A systematic review and meta-analysis. JAMA, 2011; 306: 2704-14

16. Teng R, Oliver $\mathrm{S}$, Hayes MA et al: Absorption, distribution, metabolism, and excretion of ticagrelor in healthy subjects. Drug Metab Dispos, 2010; 38: 1514-21

17. Krishna V, Diamond GA, Kaul S: Do platelet function testing and genotyping improve outcome in patients treated with antithrombotic agents?: The role of platelet reactivity and genotype testing in the prevention of atherothrombotic cardiovascular events remains unproven. Circulation, 2012; 125: 1288-303

18. Yamaguchi $Y$, Abe $T$, Sato $Y$ et al: Effects of VerifyNow P2Y12 test and CYP2C19*2 testing on clinical outcomes of patients with cardiovascular disease: A systematic review and meta-analysis. Platelets, 2013; 24: 352-61

19. Breet NJ, van Werkum JW, Bouman HJ et al: Comparison of platelet function tests in predicting clinical outcome in patients undergoing coronary stent implantation. JAMA, 2010; 303: 754-62

20. Al-Jenoobi Fl, Alkharfy KM, Alghamdi AM et al: CYP2C19 genetic polymorphism in Saudi Arabians. Basic Clin Pharmacol Toxicol, 2013; 112: 50-54

21. Pang YS, Yang YS, Wong LP et al: Genetic polymorphism of cytochrome P450 2C19 in healthy Malaysian subjects. Br J Clin Pharmacol, 2004; 58: 332-35 
22. Yamada S, Onda M, Kato $S$ et al: Genetic differences in CYP2C19 single nucleotide polymorphisms among four Asian populations. J Gastroenterol, 2001; 36: 669-72

23. Favelamendoza AF, Martinezcortes G, Hernandezzaragoza M et al: Genetic variability of CYP2C19 in a Mexican population: Contribution to the knowledge of the inheritance pattern of CYP2C19*17 to develop the ultrarapid metabolizer phenotype. J Genet, 2015; 94: 1-5

24. Kubota T, Chiba K, Ishizaki T: Genotyping of S-mephenytoin 4'-hydroxylation in an extended Japanese population. Clin Pharmacol Ther, 1996; 60: 661-66

25. Ding Y, Xu D, Zhang $X$ et al: Genetic polymorphisms and phenotypic analysis of drug-metabolizing enzyme CYP2C19 in a Li Chinese population. Int J Clin Exp Pathol, 2015: 8: 13201-8

26. Mcgraw J, Waller D: Cytochrome P450 variations in different ethnic populations. Expert Opin Drug Metab Toxicol, 2012; 8: 371-82

27. Rehman KU, Akhtar T, Sabar MF et al: Allele frequency distribution of CYP2C19*2 allelic variants associated with clopidogrel resistance in cardiac patients. Exp Ther Med, 2015; 10: 309-15

28. Scordo MG, Caputi AP, D'Arrigo C et al: Allele and genotype frequencies of CYP2C9, CYP2C19 and CYP2D6 in an Italian population. Pharmacol Res, 2004; 50: 195-200

29. Horai Y, Nakano M, Ishizaki T et al: Metoprolol and mephenytoin oxidation polymorphisms in Far Eastern Oriental subjects: Japanese versus mainland Chinese. Clin Pharmacol Ther, 1989; 46: 198-207

30. Sukasem C, Tunthong R, Chamnanphon M et al: CYP2C19 polymorphisms in the Thai population and the clinical response to clopidogrel in patients with atherothrombotic-risk factors. Pharmgenomics Pers Med, 2013; 6: 85-91

31. Luo HR, Poland RE, Lin KM, Wan YJ: Genetic polymorphism of cytochrome P450 2C19 in Mexican Americans: A cross-ethnic comparative study. Clin Pharmacol Ther, 2006; 80: 33-40

32. Gaikovitch EA, Cascorbi I, Mrozikiewicz PM et al: Polymorphisms of drug metabolizing enzymes CYP2C9, CYP2C19, CYP2D6, CYP1A1, NAT2 and of P-glycoprotein in a Russian population. Eur J Clin Pharmacol, 2003; 59: $303-12$
33. He N, Yan FX, Huang SL et al: CYP2C19 genotype and S -mephenytoin 4'-hydroxylation phenotype in a Chinese Dai population. Eur J Clin Pharmacol, 2002; 58: 15-18

34. Xiao ZS, Goldstein JA, Xie HG et al: Differences in the incidence of the CYP2C19 polymorphism affecting the S-mephenytoin phenotype in Chinese $\mathrm{Han}$ and Bai populations and identification of a new rare CYP2C19 mutant allele. J Pharmacol Exp Ther, 1997; 281: 604-9

35. Aynacioglu AS, Sachse C, Atilla Bozkurt MD et al: Low frequency of defective alleles of cytochrome P450 enzymes 2C19 and 2D6 in the Turkish population. Clin Pharmacol Ther, 1999; 66: 185-92

36. Chang M, Dahl ML, Tybring G et al: Use of omeprazole as a probe drug for CYP2C19 phenotype in Swedish Caucasians: Comparison with S-mephenytoin hydroxylation phenotype and CYP2C19 genotype. Pharmacogenetics, 1995; 5: 358-63

37. Bravo-Villalta HV, Yamamoto K, Nakamura K et al: Genetic polymorphism of CYP2C9 and CYP2C19 in a Bolivian population: An investigative and comparative study. Eur J Clin Pharmacol, 2005; 61: 179-84

38. Halling J, Petersen MS, Damkier P et al: Polymorphism of CYP2D6, CYP2C19, CYP2C9 and CYP2C8 in the Faroese population. Eur J Clin Pharmacol, 2005; 61: 491-97

39. Roh HK, Dahl ML, Tybring G et al: CYP2C19 genotype and phenotype determined by omeprazole in a Korean population. Pharmacogenetics, 1996; 6: $547-51$

40. Goldstein JA, Ishizaki T, Chiba K et al: Frequencies of the defective CYP2C19 alleles responsible for the mephenytoin poor metabolizer phenotype in various Oriental, Caucasian, Saudi Arabian and American black populations. Pharmacogenetics, 1997; 7: 59-64

41. Herrlin K, Massele AY, Jande M et al: Bantu Tanzanians have a decreased capacity to metabolize omeprazole and mephenytoin in relation to their CYP2C19 genotype. Clin Pharmacol Ther, 1998; 64: 391-401

42. Persson I, Aklillu E, Rodrigues F et al: S-mephenytoin hydroxylation phenotype and CYP2C19 genotype among Ethiopians. Pharmacogenetics, 1997; 6: 521-26

43. Masimirembwa C, Bertilsson L, Johansson I et al: Phenotyping and genotyping of S-mephenytoin hydroxylase (cytochrome P450 2C19) in a Shona population of Zimbabwe. Clin Pharmacol Ther, 1995; 57: 656-61 